\title{
Vergleichende morphometrische Untersuchungen an der Gartenspitzmaus aus Jugoslawien
}

\author{
Indulis VESMANIS
}

\begin{abstract}
Vesmanis I., 1976: Vergleichende morphometrische Untersuchungen an der Gartenspitzmaus aus Jugoslawien. Acta theriol. 21, 35: 513-526 [Mit 3 Tabellen \& 8 Abb.].

In Jugoslawien kommen zwei Unterarten von Crocidura suaveolens (P a $11 \mathrm{a} \mathrm{s}, 1811)$ vor: mimula in Kroatien und Istrien und balcanica in Mittel- und Süd-Jugoslawien. An Hand einer Aufsammlung wird diese Tatsache überprüft. Es wird mit Tabellen und Abbildungen nachgewiesen, daß C. suaveolens von Norden nach Süden zu größer wird.

[Forschungsinst. Senckenberg, 25 Senckenberganlage, Frankfurt/M-1, BRD].
\end{abstract}

\section{EINLEITUNG}

Nach O ndrias (1970) kommen in Jugoslawien zwei Unterarten von Crocidura suaveolens vor: 1. C. s. mimula - er nennt die Fundorte Medveja und Rovinij (Istrien) - und 2. C. s. balcanica, eine neue Unterart, die er selbst von Griechenland (loc. typ: Kryonerion, Attica) beschreibt. Für balcanica werden die Fundorte Ohrid, Dubrovnik, Sarajevo, Biograd, Polienik und Zadar in Jugoslawien genannt. Als wichtigstes Kriterium zur Unterscheidung der beiden Unterarten wird die Condylobasal-Länge angeführt. Die Condylobasal-Länge (CBL) bei mimula soll von $15.4-17.4 \mathrm{~mm}$ reichen, im Durchschnitt aber weniger als $16.6 \mathrm{~mm}$ sein. Bei balcanica soll die CBL $18.5 \mathrm{~mm}$ erreichen und im Durchschnitt mehr als $16.9 \mathrm{~mm}$ aufweisen.

Eine umfangreiche Aufsammlung von suaveolens aus Istrien, Kroatien, Bosnien, Dalmatien und Makedonien (Jugoslawien) soll in der vorliegenden Arbeit morphometrisch dargestellt werden. Die Tiere werden mit kleinen Populationen aus Italien, Griechenland, S-Deutschland und Österreich verglichen. Da der systematische Status der einzelnen Unterarten in diesem Raum recht undurchsichtig ist (M ille r, 1912; Chaworth-Musters, 1932; W olf, 1958; Niet h a m mer, 1962, Wit te, 1964; K a h mann, 1964; S pitze n berger, 1970 und W a g$n$ e r, 1974) wurden die einzelnen Unterarten zunächst bei der Darstellung nicht beachtet. 


\section{MATERIAL}

C. suaveolens (Jugoslawien): Medveja, $10 \mathrm{~km} \mathrm{s.} \mathrm{Opatija,} \mathrm{Istrien;} \mathrm{7.X.1964;} q$ (Schädel, Balg): SMF 23442. Jamarice bei Novska, Kroatien; 1.-3.I., 16., 22.-23.ViIT. 1965, 9.X.1966; $2 \sigma^{7} \sigma^{7}, 9$ ㅇ (11 Schädel, 11 Bälge): SMF 40510-520. Gornja Kustosija, Zagreb, Kroatien; 28.XII.1964, 13., 30.IX., 14.X.1965, 6.I., 15.X.1966; 1б, 6우, 3 sex? (10 Schädel, 10 Bälge): SMF 40500-509. Mosor-Gebirge, Dalmatien; 17.VII. 1960; † (Schädel, Balg): SIVF 45573. Sinj, Dalmatien; 24., 30.VII.1960; 4 o ㅇ (4 Schädel, 4 Bälge): SMF 45569-572. Zwischen Rovanjska und Policnik, $15 \mathrm{~km}$ NO Zadar, Dalmatien; 26.IX.1964; of (Schädel, Balg): SMF 23440. $1 \mathrm{~km}$ SW Policnik, $10 \mathrm{~km}$ von Zadar, Dalmatien; 27.IX.1964; o (Schädel, Balg): SMF 23441. Dálmatinische Küste bei Biograd, Hinterland des Vrana Sees, Dalmatien; 4.IV.1961; of (Schädel, Balg): SMF 19589. Ohrid, Biolcgische Station, Makedonien; 17., 18.IX. 1964; $5 \sigma^{\top} \sigma^{7}, 1$ ㅇ (6 Schädel, 6 Bälge): SMF 23611-616. Sarajevo, Bosnien; 26.VI 1962; (Balg): NKB 66.337. Ombla-Tal, Dubrovnik; 7., 9.VI.1962; of, of (2 Schädel, 2 Bälge): MKB 66.335-336.

\section{Vergleichsmaterial}

C. suaveolens (Peleponnes, Griechenland): Zacharo; 5.IV.1958; 2 sex? (2 Schädel, 2 Alkohol)! SMF 26796, 26802. Loutra Kaiaisha; 4.IX.1958; 1 sex? (Schädel, Alkohol): SMF 26797. Kavassila; 8.IX.1958; 우 (Schädel, Balg): SMF 26783. Olympia; 7.IX.1958; ơ (Schädel, Balg): SMF 26782.

C. suaveolens (Italien): Calliano, Trento; 11., 14.VIII.1962; $+2,2 \sigma^{\top} \sigma^{\top}$ (3 Schädel, 3 Bälge): SMF 20784-786. Peschici, Mte. Gargano; 20.VIII.1961; † (Schädel, Balg): MKB 66.338. Javoliere di Puglia, Nite. Gargano; 13.VI.1961; ㅇ (Schädel, Balg); MKB:,66.339. Ischitella, Mte. Gargano; 23.V.1961; † (Schädel, Balg): MKB 66.340. Gli, Astroni, Neapol; 26.IX.1958; 우 (Schädel, Balg): MKB 58.209.

C. suaveolens (SBR-Deutschland): Bergheim, Donau; 13.III.1950; ơ (Schädel, Balg): SKM 1088. Unterstall, Donau; 21., 27.XII.1949; 6.I.1950; $5 \sigma^{\top} \sigma^{\top}$ (5 Schädel, 5 Bälge): SKM 908-10, 915, 929. Rimsting, Oberbayern; 27.XII.1949; 21.XI.1950; ơ , ㅇ (1 Schädel, 2 Bälge): SKM 911; 1466. Dingolfing, Oberbayern; 3., 17.-18., 21.X.1949; 55. ऊ7, 1 ㅇ (6 Schädel, 6 Bälge): SKM 795-97; 819-20; 865. Straubing, Niederbayern; 21.IV.1950; ㅇ (Schädel, Balg): SKM 1124. Erching, Oberbayern; 22.IX.1950; f (Balg): SKM 1428. Pottenstein, Mittelfranken; 14.XI.1950; ㅇ (Schädel, Balg): SKM 1457. Kleinlangheim, Unterfranken; 12.II.1956; 2 우 (2 Bälge): SKM 2157, 2974. Rergen, Oberbayern; IX.1944; \& (Schädel, Balg): SKM 68. Buchdorf, Schwaben; 27.Xİ., 8., 15.XII.1950; $3 \sigma^{\top} \sigma^{\top}$ (3 Schädel, 3 Bälge): SKM 1475a, 1492, 1511. Klafferstraß, Niederbayern; 6.XI.1951; ơ (Schädel, Balg): SKM 1787. Breitenbrunn, Oberfranken; 8.X.1951; $\sigma^{\top}$ (Schädel, Balg): SKM 1770. Wülfershausen, Unterfranken; 10.VIII.1951; 20.X.1951; 2 우 (2 Schädel, 2 Bälge): SKM 1413, 1780.

C. suaveolens (Österreich): St. Gilgen, Salzburg; 16., 24.XI., 3.XII.1944; 11.I.1945; 15, 4 우우 (5 Schädel, 5 Bälge): SKM 11, 16, 18, 59-60. Weissenbach; 24.X.1945; 운 (Schäđel, Balg): SKM 97.

\section{ABKÜRZUNGEN}

(a). Körperabmessungen: TL-Total-Länge; KR-Kopf-Rumpf-Länge; S-Schwanz-Länge; HF-Hinterfuß-Länge, gemessen ohne Krallen.

(b) Schädelabmessungen: $1-\mathrm{CIL}=$ Condylobasal-Incisiv-Länge; $2-\mathrm{CBL}=\mathrm{Condy}-$ lobasal-Länge; 3-PL=Prosthio-Lambda; 4-MSQ= Maxillofrontale-Squamosum; 
5-SKB $=$ Schädelkapsel-Breite; 6- $\mathrm{IB}=$ Interorbital-Breite; 7-JB=Joch-Breite; 8-$-\mathrm{AB}=$ Anteorbital-Breite; $9-\mathrm{SKL}=$ Schädelkapsel-Länge; $10-\mathrm{GL}=$ Gaumen-Länge; $11-\mathrm{SB}=$ Staphylion - Basion; $12-\mathrm{SV}=$ Sphenobasion - Vertex $13-M^{2}-M^{2}=$ maximale

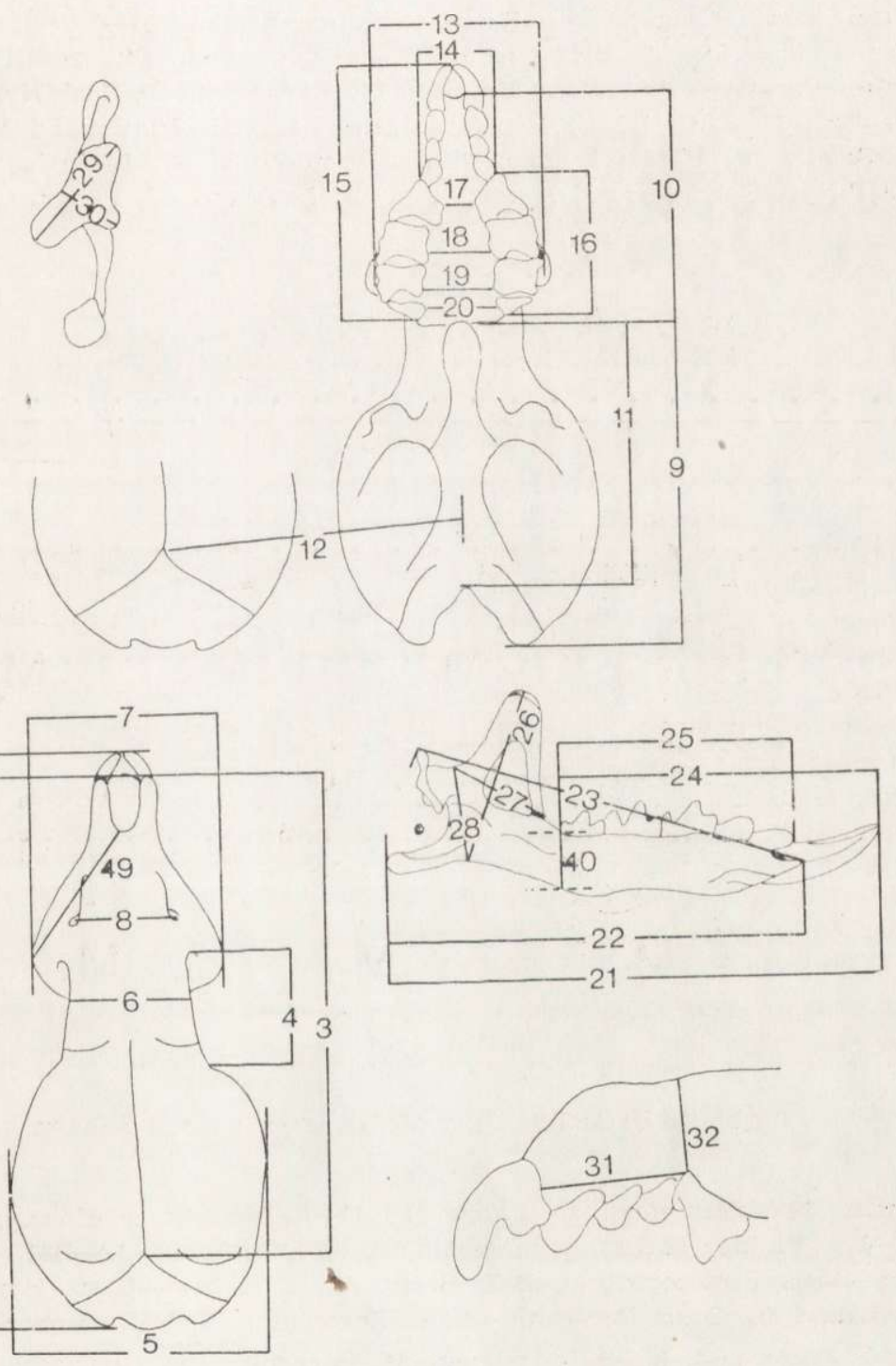

Abb. 1. Darstellung der Meßstrecken am Schädel.

Ereite über $M^{2}$, gemessen an den Kronen; $14-\mathrm{RB}=$ rostrale Breite, gemessen an den Alveolen des 3. kleinen einspitzigen Zahnes; 15-OZL=obere Zahnreihen-Länge, $I-M^{3} ; 16-\mathrm{P}^{4}-\mathrm{M}^{3}=$ Zahnreihen-Länge, $\mathrm{P}^{4}-\mathrm{M}^{3} ; 17-\mathrm{iP}^{4}-\mathrm{I} \mathrm{P}^{4}=$ innerer Abstand $\mathrm{zwi}-$ 
schen den $P^{4} ; 18-\mathrm{iM}^{1}-\mathrm{iM}^{1}=$ innerer Abstand zwischen $M^{1} ; 19-\mathrm{iM}^{2}-\mathrm{iM}^{2}=$ innerer Abstand zwischen $M^{2} ; 20-\mathrm{iM}^{3}-\mathrm{iM}^{3}=$ innerer Abstand zwischen $M^{3} ; 21-\mathrm{AI}=\mathrm{Angu}-$ lare-incl. Incisivus; 22-UKL $=$ Unterkiefer-Länge; 23- $\mathrm{CL}=$ Condylar-Länge; 24$\mathrm{UZL}=$ untere Zahnreihen-Länge, $I-M_{3} ; 25-\mathrm{C}-\mathrm{M}_{3}=$ untere Zahnreihen-Länge, $C-M_{3}$; $26-\mathrm{CH}=$ Coronar - Höhe; $27-\mathrm{CB}=$ Coronar-Breite; $28-\mathrm{PCH}=$ Postcoronar - Höhe; $29-$ GKL $=$ Gelenkkopf-Länge; $30-\mathrm{GKB}=$ Gelenkkopf-Breite; $31-\mathrm{RL}=$ Rostrum-Länge, zwischen $I$ und $P^{4} ; 32-\mathrm{RH}=$ rostrale Höhe über dem $P^{4} ; 33-\mathrm{LP}^{4}=$ Länge $P^{4}$, Vorderrand Parastyl-Hinterrand Metastyl; $34-\mathrm{BP}^{4}$, Breite $\mathrm{P}^{4}$, Basis Hypoconus-Metastyl außen; 35-LM ${ }^{1}=$ Länge $M^{1}$, Vorderrand Parastyl-Hinterrand Metastyl; 36 $-\mathrm{BM}^{1}=$ Breite $M^{1}$, Parastyl außen-Basis Protoconus; $37-\mathrm{LM}^{2}=$ Länge $M^{2}$, Vorderrand Parastyl-Hinterrand Metastyl; 38- $\mathrm{BM}^{2}=$ Breite $M^{2}$, Parastyl außen-Basis

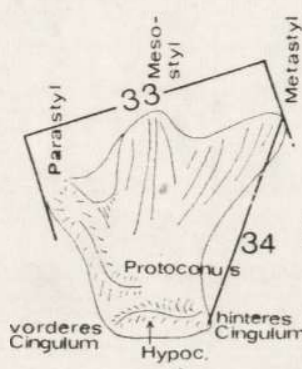

$\mathrm{P}_{4}$

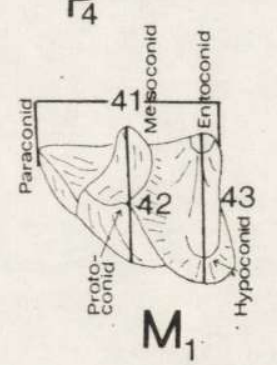

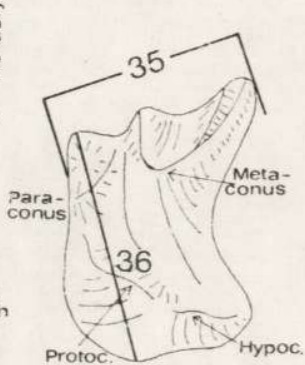

$\mathrm{M}_{1}$

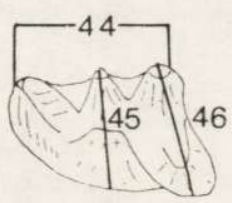

$\mathrm{M}_{2}$

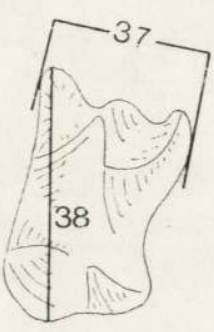

$\mathrm{M}_{2}$

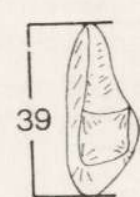

$\mathrm{M}_{3}$

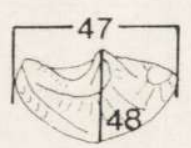

$\mathrm{M}_{3}$

Abb. 2. Darstellung der Meßstrecken an den Zähnen.

Protoconus; $39-\mathrm{LM}^{3}=$ maximale Länge $M^{3} ; 40-\mathrm{RAH}=$ Ramus-Höhe, gemessen am $\mathrm{M}_{3} ; 41-\mathrm{LM}_{1}=$ Länge $M_{1}$, Basis Paraconid-Basis Entoconid; $42-\mathrm{BM}_{1}=$ Breite $M_{1}$, Mesonid-Basis Protoconid; $43-\mathrm{BM}=$ Breite $M_{1}$, Entoconid-Basis Hypoconid; $44-$ - $\mathrm{LM}_{2}=$ Länge $M_{2}$, Basis Paraconid-Basis Entoconid; $45-\mathrm{BM}_{2}=$ Breite $M_{2}$, Mesoconid-Basis Protoconid; $46-\mathrm{BM}_{2}^{+}=$Breite $M_{2}$, Entoconid-Basis Hypoconid; $47-\mathrm{LM}_{3}=$ =Länge $M_{3} ; 48-\mathrm{BM}_{3}=$ Breite $M_{3} ; 49-\mathrm{RZ}=$ Rhinion - Zygion.

(c) andere Abkürzungen:

MKB - Museum Alexander Koenig Bnnn

SMF - Senckenberg Museum Frankfurt am Main

SKM - Sammlung Kahmann München 


\section{ERGEBNIS UND DISKUSSION}

1. Vergleich von $\mathbf{C}$. suoveolens aus Istrien, Kroatien, Dalmatien und Makedonien untereinander (Tab. 1)

Insgesamt lagen zur Untersuchung 36 C. suaveolens aus Jugoslawien vor; davon fielen auf Istrien und Kroatien 22, Dalmatien 8 und Makedonien 6 Exemplare. In Tabelle 1 sind alle Maße (Minimum, Maximum und Mittelwert) zum Vergleich angegeben. In den Schädel-Maßen CIL, CBL, PL, MSQ, SB, $\mathrm{M}^{2}-\mathrm{M}^{2}, \mathrm{OZL}, \mathrm{P}^{4}-\mathrm{M}^{3}, \mathrm{iP}^{4}-\mathrm{iP}^{4}, \mathrm{iM}^{1}-\mathrm{iM}^{1}, \mathrm{iM}^{2}-\mathrm{iM}^{2}$, $\mathrm{iM}^{3}-\mathrm{iM}^{3}, \mathrm{AI}, \mathrm{CL}, \mathrm{UZL}, \mathrm{C}-\mathrm{M}_{3}, \mathrm{CB}, \mathrm{PCH}, \mathrm{LM}^{2}, \mathrm{BM}^{2}, \mathrm{RAH}$ und $\mathrm{RZ}$ ist ein Größenzuwachs, teilweise sehr gering, von Norden nach Süden festzustellen. Dieses Zunehmen ist hier auf den Mittelwert bezogen, denn im Minimum und Maximum halten sich die Meßwerte manchmal die Waage. Es ist hier nicht zu leugnen, daß die untersuchte Population aus Istrien und Kroatien - nach Ondrias (1970) s. mimula - die kleinsten Exemplare aufweist, doch dürfte es wegen der großen Überschneidung der einzelnen Meßwerte sehr schwierig sein, eine Grenze zwischen den Unterarten mimula und balcanica durch N-Jugoslawien zu ziehen. Nach der vorliegenden Tabelle 1 scheinen wir es eher mit einem Klin im Sinne von De Lattin (1967) zu tun zu haben (vgl. auch Vesmanis, 1975). Morphologisch zeigen die Tiere untereinander aus dem Norden und Süden keinen Unterschied, auch nicht in der Form des Condylus vom Unterkiefer, der sehr variabel in seiner Gestaltung ist.

Da die von Norden nach Süden zunehmende Schädelgröße bei der sehr ungleichen Serie (bezüglich der Anzahl der Individuen) teilweise nur wenige Zehntel Milimeter ausmacht, sehe ich bei dem augenblicklichen Stand der Untersuchungen über Crocidura keinen Grund für die Berechtigung von zwei Unterarten von C. suaveolens in Jugoslawien.

2. Vergleich von $\mathbf{C}$. suaveolens aus Jugoslawien mit Tieren

aus Griechenland BR-Deutschland, Ósterreich und Italien (Tab. 2-3)

Vergleicht man die jugoslawische Serie mit Tieren von der Peleponnes, so sind die griechischen Exemplare kleiner als die vorliegenden Stücke aus Dalmatien und Makedonien, aber etwas größer als die Population aus Istrien und Kroatien. Größenmäßig müßten sie also zwischen Kroatien und Dalmatien eingestuft werden. Ondria s (1970: 379) gibt für balcanica als CBL einen Wert von 16.9 (= Mittelwert) an. Die vorliegenden Tiere von der Peleponnes haben einen Mittelwert von $17.0 \mathrm{~mm}$, sie würden also mit einem Zehntel im Bereich von balcanica liegen! $\mathrm{Ob}$ dieses Zehntel für eine Klassifizierung zur Unterart ausreicht, sei im Moment dahingestellt. 
Tabelle 1

Messdaten von Crocidura suaveolens aus Jugoslawien.

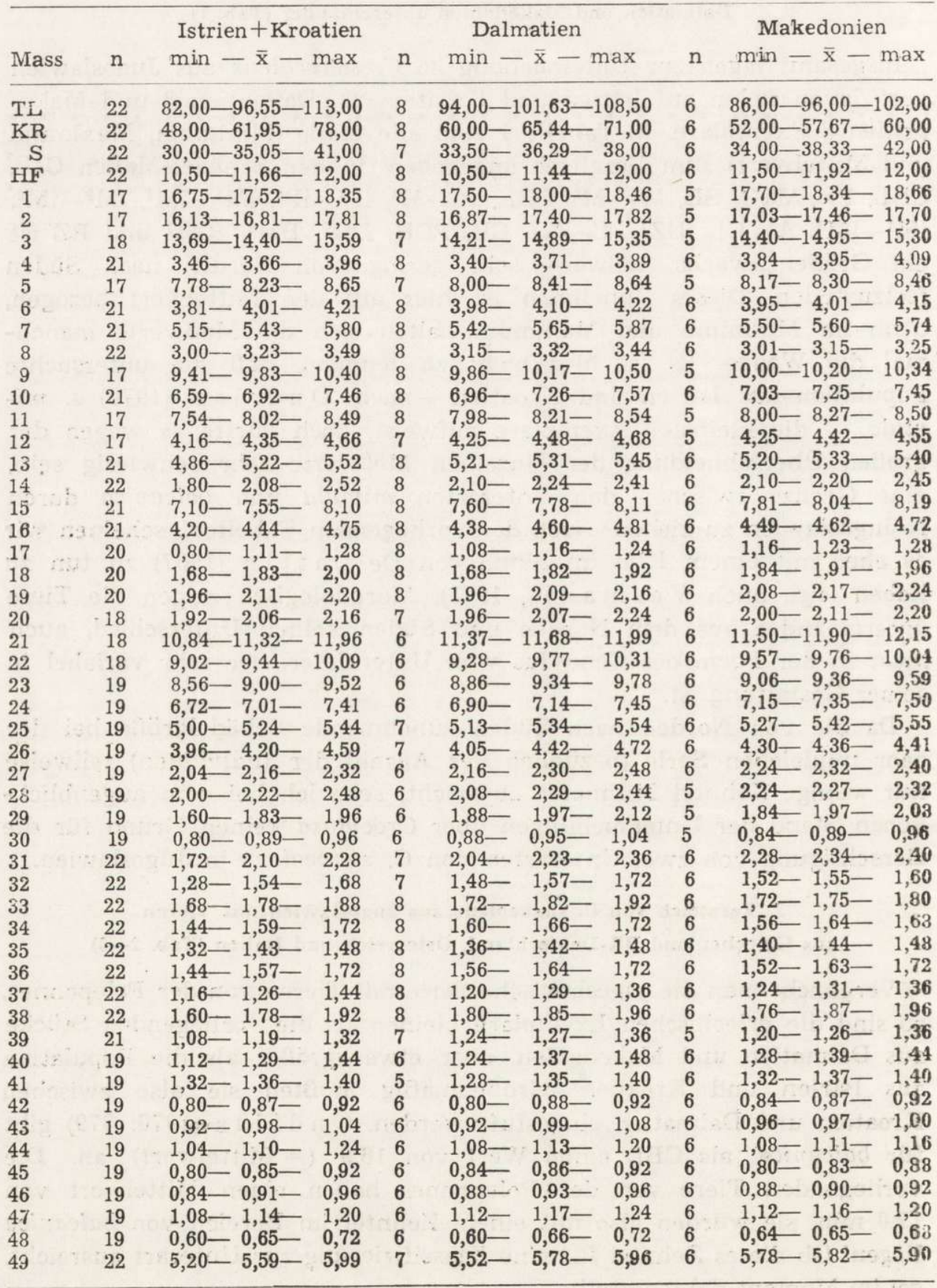


Tabelle 2

Messdaten von Crocidura suaveolens aus Bundesrepublik Deutschland, Osterreich und Italien.

\begin{tabular}{|c|c|c|c|c|c|c|c|c|c|c|}
\hline \multirow{2}{*}{$\begin{array}{l}\text { Mass } \\
\text { TL }\end{array}$} & $\mathrm{n}$ & \multicolumn{2}{|c|}{$\begin{array}{l}\text { BR-Deutschland } \\
\min -\bar{x}-\max \end{array}$} & $\mathrm{n}$ & \multicolumn{2}{|c|}{$\begin{array}{l}\text { Ósterreich } \\
\operatorname{lin}-\overline{\mathrm{x}}-\max \end{array}$} & \multicolumn{4}{|c|}{ Italien } \\
\hline & 28 & $88,00-99,32-1$ & 108,00 & 6 & $88,00-96,33-1$ & 100,00 & 7 & $00-10$ & $100,60-1$ & 99,00 \\
\hline KR & 28 & $55,00-63,43-$ & 70,00 & 6 & $57,00-62,33-$ & 65,00 & 7 & $56,00-$ & 63,14 & 69,00 \\
\hline $\mathrm{S}$ & 28 & $32,00-35,82-4$ & 41,00 & 6 & $33,00-34,33-3$ & 37,00 & 7 & $33,00-3$ & $37,43-4$ & 42,00 \\
\hline $\mathrm{HF}$ & 28 & $10,00-11,23-$ & 12,00 & 6 & $11,00-11,67-$ & 12,00 & 7 & $11,00-$ & $11,36-$ & 12,60 \\
\hline 1 & 16 & $16,00-17,34-$ & 18,30 & 6 & $16,80-17,31-$ & 17,70 & 5 & 17,56 & $17,92-$ & 18,21 \\
\hline 2 & 17 & $15,20-16,64-$ & 17,56 & 6 & $15,86-16,59-$ & 17,10 & 5 & $16,98-$ & $17,32-$ & 17,79 \\
\hline 3 & 19 & $12,77-14,11-$ & 14,92 & 6 & $13,10-14,12-$ & 14,80 & 5 & $14,54-$ & $14,93-$ & 15,20 \\
\hline 4 & 21 & $3,70-3,71-$ & 4,00 & 6 & $3,84-4,02-$ & 4,21 & 5 & $3,60-$ & $3,78-$ & 3,95 \\
\hline 5 & 18 & $7,70-8,28-$ & 8,60 & 6 & $7,80-8,13-$ & 8,42 & 5 & $7,96-$ & $8,26-$ & 8,53 \\
\hline 6 & 19 & $3,90-4,02-$ & 4,15 & 6 & $3,56-3,67-$ & 3,90 & 5 & $3,92-$ & 4,04 & 4,14 \\
\hline 7 & 22 & $5,05-5,51-$ & 5,85 & 6 & $5,06-5,44-$ & 5,70 & 6 & $5,45-$ & $5,59-$ & 5,94 \\
\hline 8 & 22 & $3,00-1,14-$ & 3,30 & 6 & $3,06-3,16-$ & 3,30 & 6 & $3,13-$ & $3,26-$ & 3,33 \\
\hline 9 & 16 & $9,06-9,64-$ & 10,10 & 6 & $9,30-9,60-$ & 9,74 & 5 & $9,67-$ & $9,92-$ & 10,20 \\
\hline 10 & 2 & $6,69-7.04-$ & 7,52 & 6 & $6,56-6,98-$ & 6 & 5 & $7,11-$ & 7,49 & 8,04 \\
\hline 11 & 15 & $7,60-8,01-$ & 8,30 & 6 & $7,80-8,00-$ & 8 & 4 & $7,89-$ & $8,07-$ & 8,31 \\
\hline 12 & 15 & $4,12-4,29-$ & 4,55 & 6 & $4,06-4,23-$ & 4,42 & 5 & $4,30-$ & 4,49 & 4,62 \\
\hline 13 & 22 & $4,75-5,19-$ & 5,40 & 6 & $4,76-5,11-$ & 5,26 & 6 & $5,21-$ & $5,32-$ & 5,60 \\
\hline 14 & 23 & $2,10-2,26-$ & 2,43 & 6 & $2,08-2,24-$ & 2,33 & 7 & $2,10-$ & $2,26-$ & 2,40 \\
\hline 15 & 22 & $7,05-7,55-$ & 8,05 & 6 & $7,25-7,56-$ & 7,84 & 7 & $7,50-$ & $7,82-$ & 8,10 \\
\hline 16 & 24 & $4,08-4,30-$ & 4,64 & 6 & $4,24-4,38-$ & 4,48 & 7 & $4,40-$ & $4,58-$ & 4,75 \\
\hline 17 & 21 & $1,00-1,13-$ & 1,28 & 6 & $4-1,17-$ & 1,2 & 6 & $1,00-$ & $1,17-$ & 1,36 \\
\hline 18 & 21 & $1,72-1,82-$ & 1,96 & 6 & $1,80-1,85-$ & 1, & 6 & $1,60-$ & $1,85-$ & 2,20 \\
\hline 19 & 21 & $1,92-2,08-$ & 2,20 & 6 & $2,04-2,11-$ & 2,2 & 6 & $1,96-$ & $2,13-$ & 2,28 \\
\hline 20 & 21 & $1,92-2,04-$ & 2,24 & 6 & $1,96-2,11-$ & 2,36 & 6 & 2,04 & $2,17-$ & 2,36 \\
\hline 21 & 20 & $10,30-11,36-$ & 12,00 & 5 & $11,04-11,36-$ & 11,50 & 6 & $11,25-$ & $11,55-$ & 11,88 \\
\hline 22 & 22 & $8,50-9,44-$ & 9,90 & 5 & $9,10-9,45-$ & 9,65 & 5 & $9,31-$ & $9,61-$ & 9,94 \\
\hline 23 & 21 & $8,20-9,09-$ & 9,55 & 6 & $50-8,93-$ & 9,20 & 5 & $9,06-$ & $9,36-$ & 9,64 \\
\hline 24 & 20 & $61-6,96-$ & 7,3 & 6 & $0-6,90-$ & 7.1 & 7 & $6,86-$ & $7,17-$ & 7,39 \\
\hline 25 & 22 & $4,90-5,40-$ & 5,50 & 5 & $0-5,14$ & 5 & 7 & $5,25-$ & $5,39-$ & 5,50 \\
\hline 26 & 21 & $4,10-4,27-$ & 4,56 & 6 & $4,05-4,22-$ & 4 & 7 & $4,26-$ & $4,38-$ & 4,50 \\
\hline 27 & 20 & $2,16-2,28-$ & 2,40 & 6 & $2,28-2,30-$ & 2,36 & 7 & 2,24 & 2,30 & 2,40 \\
\hline 28 & 20 & $2,20-2,30-$ & 2,42 & 6 & $2,16-2,25-$ & 2,36 & 7 & $2,20-$ & $2,31-$ & 2,44 \\
\hline 29 & 21 & $1,76-1,88-$ & 1,96 & 5 & $1,64-1,79-$ & 1,9 & 7 & $1,76-$ & 1,94 & 2,08 \\
\hline 30 & 21 & $0,80-0,90-$ & 1,00 & 6 & $0,80-0,85-$ & 0,8 & 7. & 0,84 & $0,91-$ & 0,96 \\
\hline 31 & 21 & $2,04-2,23-$ & 2,40 & 6 & $08-2,25-$ & 2,3 & 6 & $2,32-$ & $2,37-$ & 2,48 \\
\hline 32 & 22 & $32-1,49-$ & 1,80 & 6 & $12-1,41-$ & 1,72 & 6 & $1,40-$ & $1,66-$ & 1,88 \\
\hline 33 & 22 & $1,64-1,73-$ & 1,80 & 6 & $1,68-1,71-$ & 1,72 & 7 & $1,68-$ & 1,79 & 1,83 \\
\hline 34 & 21 & $1,40-1,56-$ & 1,64 & 6 & $1,48-1,53-$ & 1,56 & 7 & $1,56-$ & 1,64 & 1,76 \\
\hline 35 & 22 & $1,24-1,37-$ & 1,44 & 6 & $1,28-1,32-$ & 1,36 & 6 & $1,32-$ & 1,43 & 1,56 \\
\hline 36 & 22 & $1,44-1,57-$ & 1,68 & 6 & $1,52-1,56-$ & 1,60 & 7 & $1,56-$ & 1,59 & 1,64 \\
\hline 37 & 22 & $1,12-1,22-$ & 1,36 & 6 & $1,12-1,15-$ & 1,16 & 6 & 1,24 & 1,30 & 1,36 \\
\hline 38 & 22 & $1,64-1,78-$ & 1,92 & 6 & $1,68-1,73-$ & 1,76 & 6 & $1,76-$ & $1,81-$ & 1,92 \\
\hline 39 & 22 & $1,12-1,25-$ & 1,32 & 6 & $1,20-1,21-$ & 1,28 & 7 & $1,16-$ & $1,22-$ & 1,32 \\
\hline 40 & 21 & $1,20-1,41-$ & 1,44 & 6 & $1,16-1,31-$ & 1,60 & 7 & 1,24 & $1,37-$ & 1,52 \\
\hline 41 & 21 & $1,20-1,31-$ & 1,40 & 6 & $1,28-1,29-$ & 1,32 & 6 & $1,32-$ & 1,36 & 1,40 \\
\hline 42 & 22 & $0,80-0,89-$ & 0,96 & 6 & $0,80-0,85-$ & 0,88 & 6 & $0,88-$ & 0,94 & 1,04 \\
\hline 43 & 22 & $0,88-0,99-$ & 1,08 & 6 & $0,88-0,93-$ & 0,96 & 6 & $0,96-$ & $1,02-$ & 1,08 \\
\hline 44 & 22 & $1,04-1,08-$ & 1,20 & 6 & $1,00-1,06-$ & 1,12 & 6 & $1,08-$ & $1,12-$ & 1,16 \\
\hline 45 & 22 & $0,80-0,87-$ & 0,92 & 6 & $0,76-0,81-$ & 0,84 & 6 & 0,84 & $0,91-$ & 1,00 \\
\hline 46 & 22 & $0,84-0,94-$ & 1,00 & 6 & $0,80-0,88-$ & 0,92 & 5 & $0,88-$ & 0,94 & 0,96 \\
\hline 47 & 22. & $1,00-1,11-$ & 1,16 & 6 & $1,08-1,09-$ & 1,12 & 6 & $1,12-$ & $1,15-$ & 1,20 \\
\hline 48 & 21 & $0,56-0,65-$ & 0,72 & 6 & $0,56-0,64-$ & 0,68 & 6 & 0,64 & $0,67-$ & 0,72 \\
\hline 49 & 18 & $5,10-5,56-$ & 5,82 & 6 & $5,46-5,65-$ & 6,00 & 7 & 5,64 & $5,78-$ & 5,87 \\
\hline
\end{tabular}


Die vorliegende Reihe aus Süd-BRD und Österreich hat deutlich die kleinsten Maße.

Die Tiere aus Italien reichen an die Exemplare aus Dalmatien und Makedonien heran, sind jedoch größer als die kleine Reihe von der Peleponnes. Hieraus folgernd könnte man nachfolgende Gliederung an Hand des vorliegenden Untersuchungsmaterials auf Unterartbasis annehmen:

Bundesrepublik Deutschland+Österreich $=$ C. $s$. mimula Jugoslawien + Griechenland $=C$. s. balcanica Italien $=C . s . \operatorname{ssp}(?)$

Tabelle 3

Messdaten von Crocidura suaveolens der Peleponnes (Griechenland).

\begin{tabular}{|c|c|c|c|c|c|c|c|}
\hline Mass & $\mathrm{n}$ & $\min$ & $-\overline{\mathrm{x}}-$ & $\max$ & Mass & $\mathrm{n}$ & $\min -\bar{x}-\max$ \\
\hline TL & 5 & $97,00-1$ & $100,00-1$ & 104,00 & 24 & 5 & $6,90-7,09-7,30$ \\
\hline KR & 5 & $57,00-$ & $60,80-$ & 65,00 & 25 & 5 & $5,07-5,29-5,45$ \\
\hline $\mathrm{S}$ & 5 & $37,00-$ & $39,20-$ & 42,00 & 26 & 5 & $4,25-4,39-4,47$ \\
\hline HF & 5 & $11,30-$ & $11,68-$ & 12,30 & 27 & 5 & $2,32-2,39-2,48$ \\
\hline 1 & 4 & $17,25-$ & 17,74 & 18,20 & 28 & 5 & $2,28-2,37-2,44$ \\
\hline 2 & 5 & $16,39-$ & $17,00-$ & 17,57 & 29 & 5 & $1,92-1,96-2,08$ \\
\hline 3 & 5 & $14,30-$ & $14,78-$ & 15,20 & 30 & 5 & $0,84-0,92-1,00$ \\
\hline 4 & 5 & 3,50 & 3,65 & 3,91 & 31 & 5 & $2,20-2,21-2,24$ \\
\hline 5 & 4 & $8,19-$ & 8,34 & 8,49 & 32 & 5 & $1,32-1,44-1,56$ \\
\hline 6 & 5 & $3,92-$ & $4,08-$ & 4,21 & 33 & 4 & $1,64-1,74-1,80$ \\
\hline 7 & 5 & $5,49-$ & 5,60 & 5,85 & 34 & 4 & $1,56-1,59-1,60$ \\
\hline 8 & 5 & $3,01-$ & $3,19-$ & 3,40 & 35 & 5 & $1,28-1,49-1,80$ \\
\hline 9 & 5 & $9,65-$ & $10,02-$ & 10,25 & 36 & 5 & $1,56-1,58-1,64$ \\
\hline 10 & 5 & $6,69-$ & $6,98-$ & 7,32 & 37 & 5 & $1,24-1,28-1,32$ \\
\hline 11 & 5 & $7,80-$ & $8,15-$ & 8,34 & 38 & 5 & $1,72-1,80-1,88$ \\
\hline 12 & 4 & $4,30-$ & $4,39-$ & 4,47 & 39 & 5 & $1,16-1,21-1,28$ \\
\hline 13 & 5 & $5,05-$ & $5,28-$ & 5,45 & 40 & 5 & $1,28-1,34-1,40$ \\
\hline 14 & 5 & $2,10-$ & $2,21-$ & 2,30 & 41 & 5 & $1,28-1,37-1,40$ \\
\hline 15 & 5 & $7,48-$ & $7,70-$ & 8,01 & 42 & 5 & $0,80-0,92-1,00$ \\
\hline 16 & 5 & 4,30 & $4,47-$ & 4,71 & 43 & 5 & $0,96-1,03-1,08$ \\
\hline 17 & 5 & $1,08-$ & $1,13-$ & 1,16 & 44 & 5 & $1,04-1,10-1,12$ \\
\hline 18 & 5 & $1,72-$ & $1,79-$ & 1,96 & 45 & 5 & $0,84-0,92-1,00$ \\
\hline 19 & 5 & 2,04 & $2,08-$ & 2,16 & 46 & 5 & $0,88-0,96-1,00$ \\
\hline 20 & 5 & 2,00 & $2,06-$ & 2,12 & 47 & 5 & $1,08-1,18-1,20$ \\
\hline 21 & 5 & $11,05-$ & $11,50-$ & 11,75 & 48 & 5 & $0,64-0,70-0,76$ \\
\hline 22 & 5 & $9,04-$ & $9,63-$ & 10,26 & 49 & 5 & $5,44-5,68-6,15$ \\
\hline 23 & 5 & $8,86-$ & $9,34-$ & 9,94 & & & \\
\hline
\end{tabular}

Es wird hier ganz bewußt für Italien $C$. $s$. ssp geschrieben, da kein Typen-Material untersucht worden ist (C. s. italica und C. $s$. debeauxi). Der Größenunterschied zwischen den italienischen und den Tieren aus Dalmatien und Makedonien ist sehr klein. Eine verbindliche Aussage bezüglich Italien kann jedoch erst nach dem Studium von dem TypenMaterial und nach der Untersuchung von viel mehr Vergleichsmaterial aus ganz Italien gemacht werden. 


\section{Vergleich an Hand von Korrelationsdiagrammen}

3.1. Condylobasal-Länge zu Schädelkapsel-Breite und Joch-Breite (Abb. 3)

Die Korrelation von SKB und JB zur CBL wurde in einem doppelten Diagramm zusammengefaßt, da sie ein ähnliches Ergebnis aufweist. Die

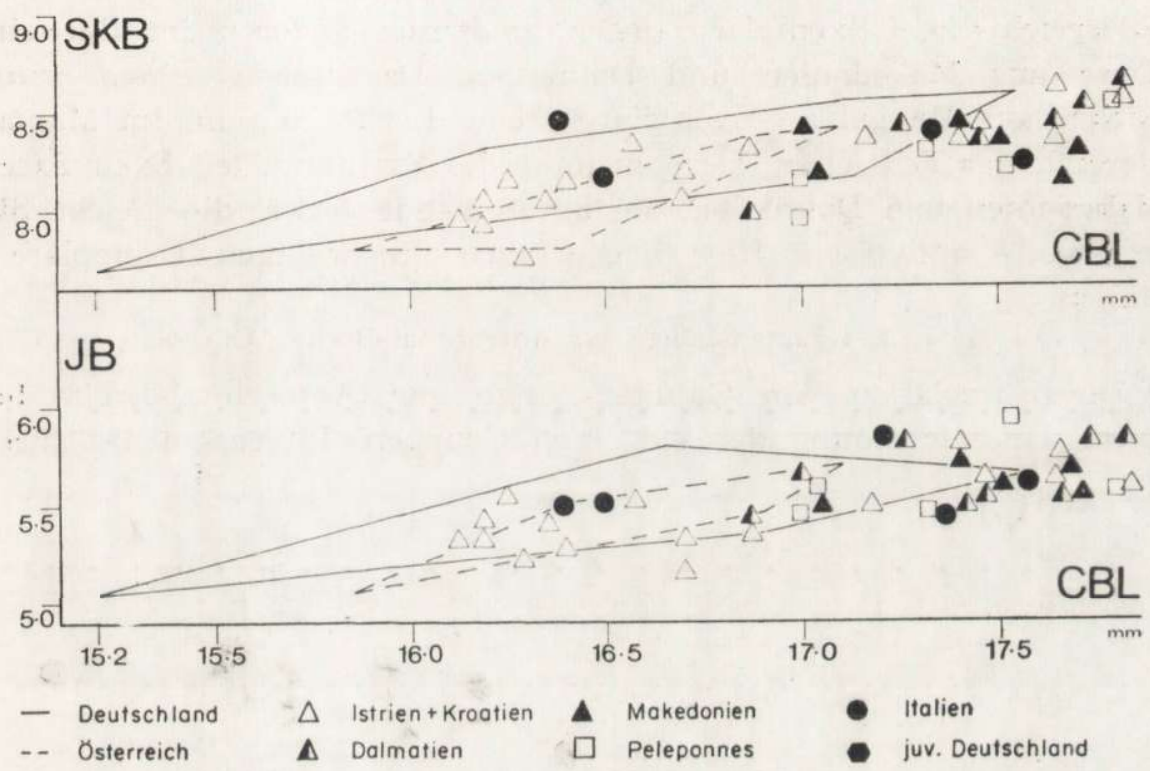

Abb. 3. Korrelationsdiagramm, Condylobasal-Länge (CBL) zu Schädelkapsel-Breite (SKB) und Joch-Breite (JB).

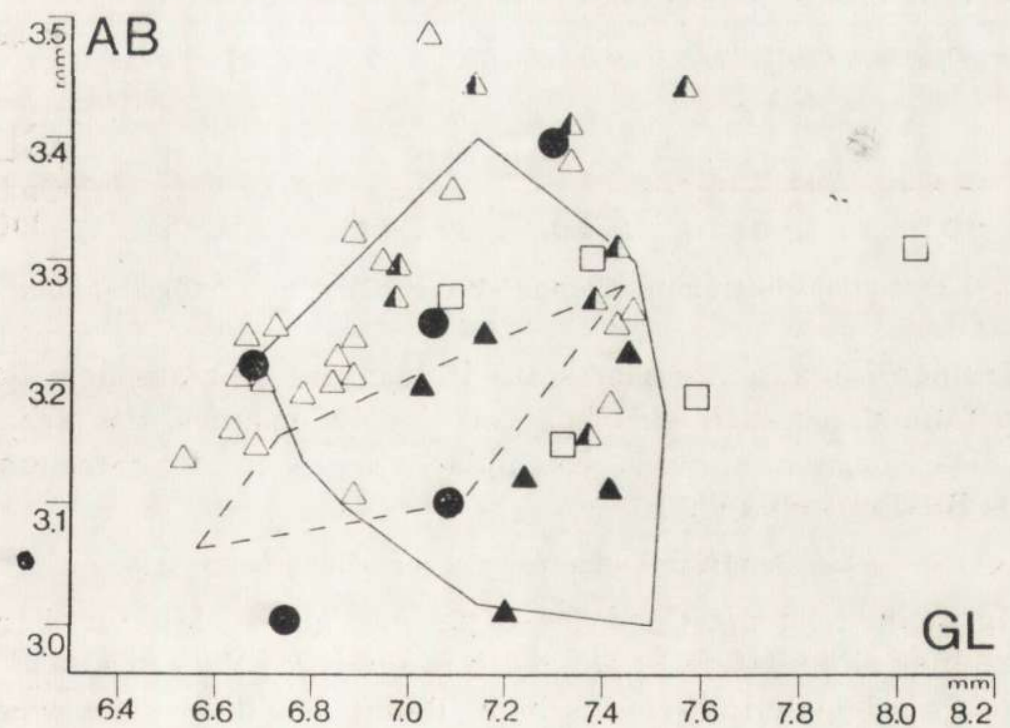

Abb. 4. Korrelationsdiagramm, Anteorbital-Breite (AB) zu Gaumen-Länge (GL). 
Tiere aus BR Deutschland zeigen die weisteste Streuung; Österreich liegt deutlich im Bereich der s-deutschen Exemplare. Tiere aus Kroatien zeigen die kleinsten Werte (bezüglich Jugoslawien), doch gehen sie in ihrer Streuung bis zu den Tieren aus Makedonien und Dalmatien hinauf. Auffallend ist, daß die Tiere von der Peleponnes einmal im Bereich der österreichischen Exemplare liegen, und zum anderen im Bereich der Tiere aus Makedonien und Dalmatien. Dazwischen scheint zunächst (sicher aus Mangel an Exemplaren) eine Lücke zu sein. Im MaximumBereich der deutschen Tiere beginnt die Streuung der Exemplare aus Makedonien und Dalmatien, die untereinander etwa die gleiche Streuungsweite aufweisen. Hier liegen auch die wenigen Exemplare aus Italien.

\subsection{Gaumen-Länge zur Anteorbital-Breite (Abb. 4)}

Die Korrelation von Gaumen-Länge und Anteorbital-Breite bringt keine Unterscheidung der einzelnen Gruppen. Interessant ist hier die

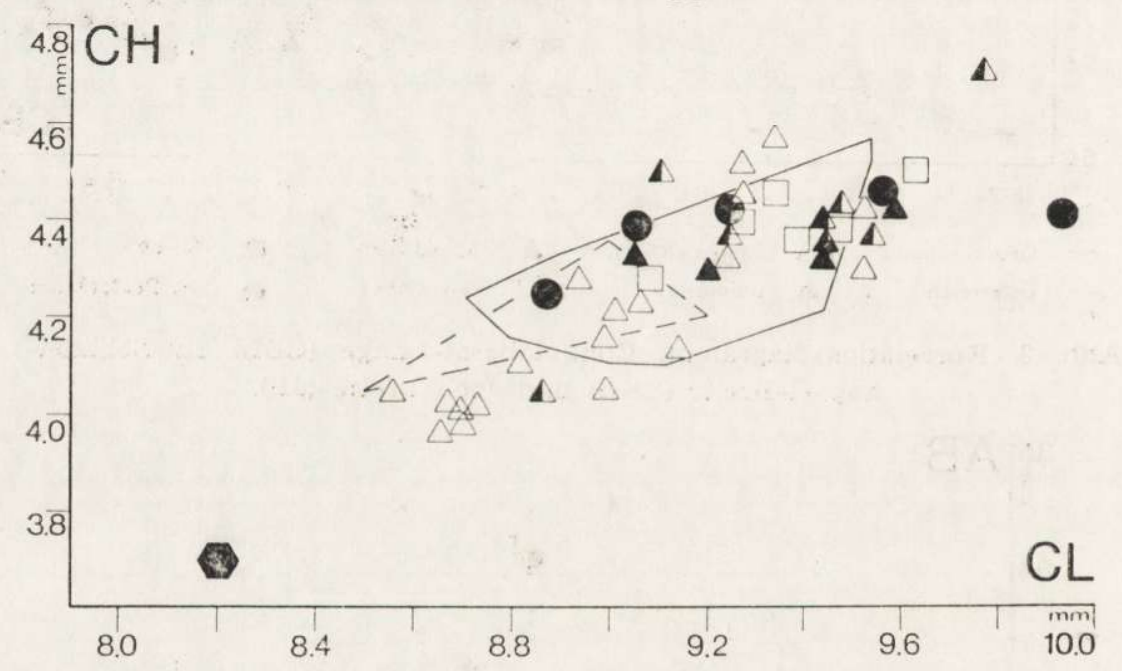

Abb. 5. Korrelationsdiagramm, Coronar-Höhe $(\mathrm{CH})$ zu Condylar-Länge (CL).

große Gaumen-Länge eines Tieres aus Italien, die ganz aus dem Rahmen fällt. Auffallend ist auch ein Tier von der Peleponnes, das sehr klein ausfällt. Die meisten anderen Exemplare liegen in der Streuung der Tiere aus BR-Deutschland.

3.3. Condylar-Länge zur Coronar-Höhe (Abb. 5)

Im allgemeinen ist die Condylar-Länge ein gutes Maß zur Trennung von einzelnen Population (vgl. Kahmann \& Vesmanis, 1975). Im jugoslawischen und griechischem Raum ist dies keineswegs der Fall. Individuen aus Kroatien streuen über die gesamte aufgezeich- 
nete CL-Länge; sie zeigen sogar teilweise kleinere Werte als Exemplare aus BR-Deutschland. Die wenigen Tiere von der Peleponnes, die nach Ondrias (1970) großwüchsig sein müßten, liegen zum größten Teil in der Streuung der mimula - Exemplare aus BR-Deutschland. Ein jugendliches Tier (keine Abkauungsspuren an den Molaren) aus BR-Deutschland zeigt einen extrem kleinen Wert auf und liegt weit abseits. Eine Trennung von kroatischen und makedonischen Tieren ist nicht genau durchführbar. Die Überlappung der Meßwerte ist auch in diesem Diagramm zu groß.

3.4. Länge $P^{4}$ zur Breite $P^{4}$ (Abb. 6)

Die Korrelation von Längen- und Breitenmaßen bei Backen-Zähnen wurde bei Kahmann \& V es man is (1974) erfolgreich durchgeführt. Die Tiere aus BR-Deutschland zeigen hier wieder die kleinsten Werte,

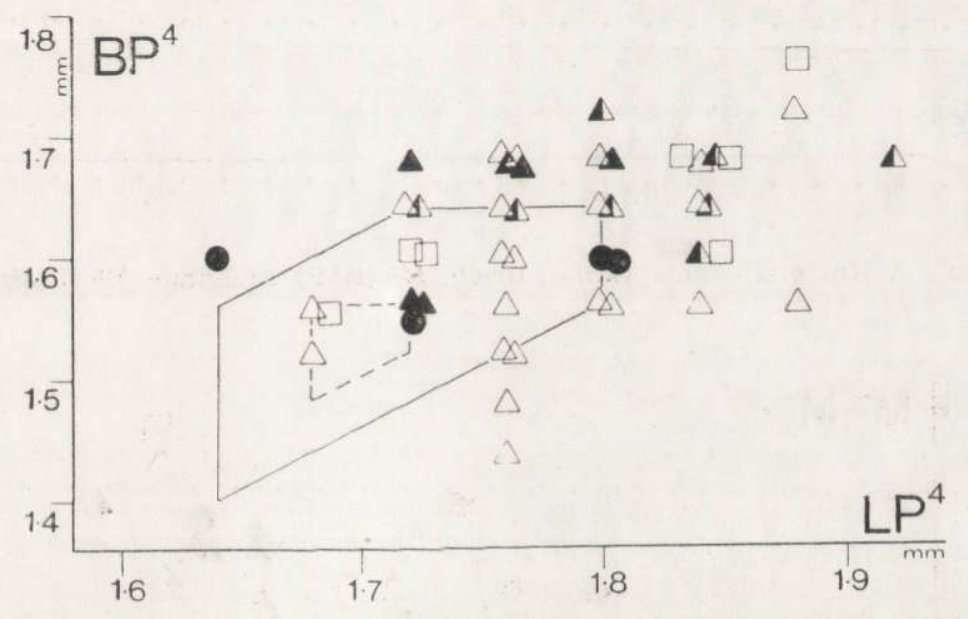

Abb. 6. Korrelationsdiagramm, Breite $P^{4}\left(\mathrm{BP}^{4}\right)$ zu Länge $P^{4}\left(\mathrm{LP}^{4}\right)$.

österreichische Exemplare liegen mitten in der Streuung der deutschen Exemplare. Auch die Tiere von der Peleponnes haben kleine $\mathrm{P}^{4}$ Abmessungen. Innerhalb von Jugoslawien lassen sich die Tiere, die von Ondrias (1970) als mimula bezeichnet worden waren, nicht eindeutig von balcanica trennen, auch nicht von den Individuen aus Italien.

3.5. Länge $M^{2}$ zu Breite $M^{2}$ (Abb. 7)

Der obere $M^{2}$ eignet sich hier noch am wenigsten zur Trennung der Unterarten. Ein Tier aus Kroatien hat einen extrem großen $\boldsymbol{M}^{2}$. Im

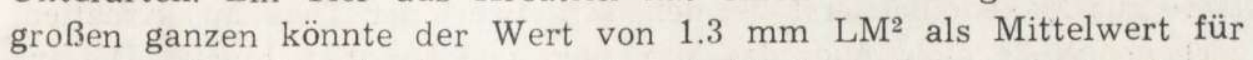
den gesamten jugoslawischen und griechischen Raum angenommen werden. Etwa $1.8 \mathrm{~mm}$ für die $\mathrm{BM}^{2}$. 
3.6. Zahnreihen-Länge $P^{4}-M^{3}$ zur maximalen Breite über $M^{2}$ (Abb. 8)

Auch bei diesem Diagramm erhalten wir ein ähnliches Ergebnis wie es uns die vorangegangenen Korrelationen gezeigt haben: die kroatischen Tiere zeigen eine recht große Streuungsweite; zwar beginnen die

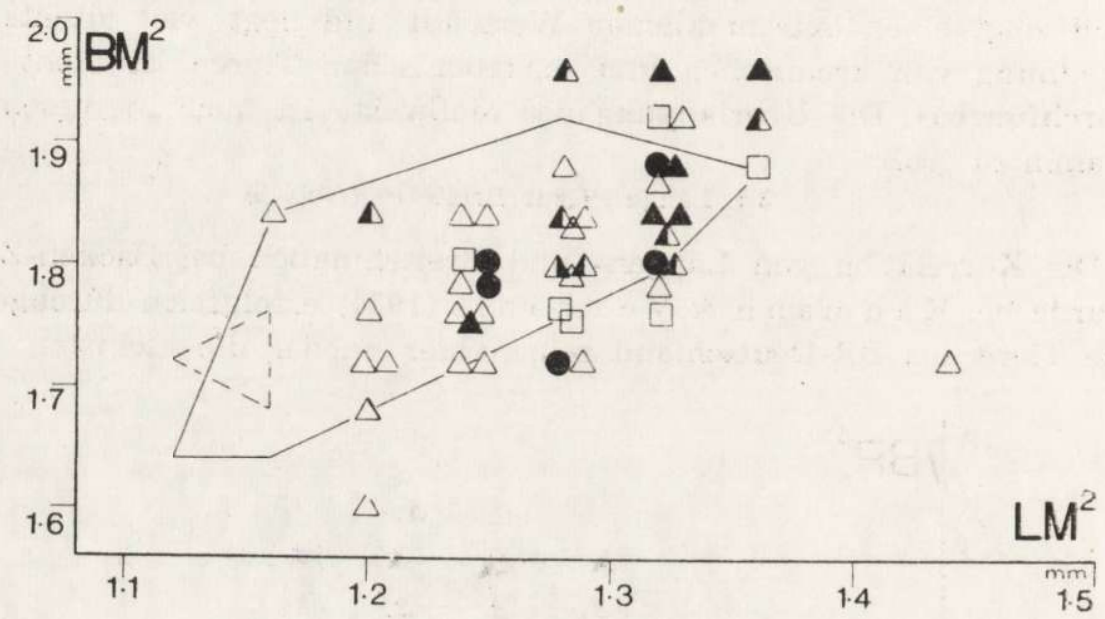

Abb. 7. Korrelationsdiagramm, Breite $M^{2}\left(\mathrm{BM}^{2}\right)$ zu Länge $M^{2}\left(\mathrm{LM}^{2}\right)$.

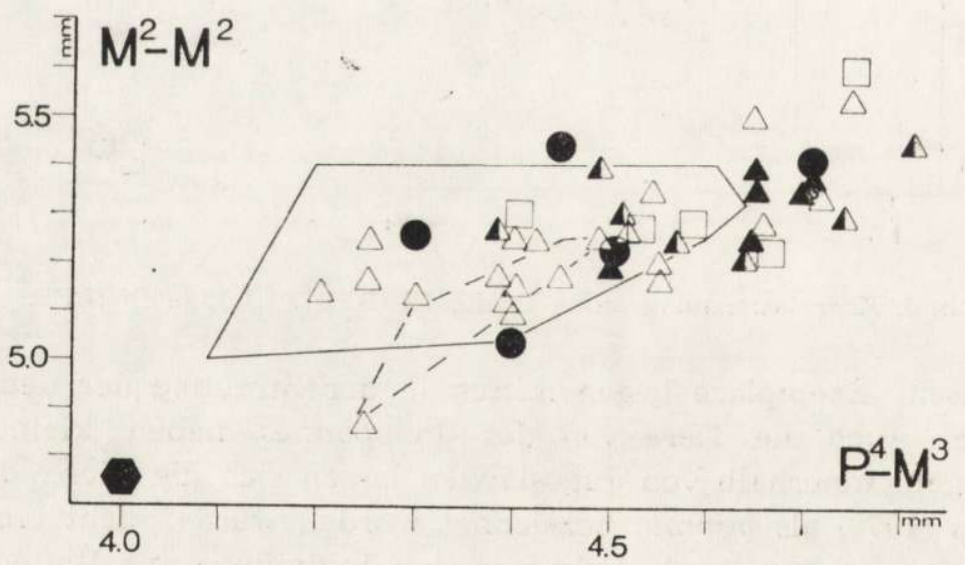

Abb. 8. Korrelationsdiagramm, maximale Breite, Kronen, über $M^{2}\left(M^{2}-M^{2}\right)$ zur Zahnreihen-Länge $P^{4}-M^{3}$.

Tiere aus Makedonien erst bei dem Mittelwert der Tiere aus Kroatien, doch gehen sie nicht über das Maximum von Kroatien hinaus. Die Individuen von der Peleponnes liegen zum großen Teil (3/4) im Bereich der deutschen Tiere. 


\section{ZUSAMMENFASSUNG DER ERGEBNISSE}

1. Tabellen und Korrelationsdiagramme zeigen, daß eine eindeutige Trennung von nord und mittel- und südjugoslawischen $C$. suaveolens auf Unterartbasis nicht möglich ist.

2. Bezüglich des Mittelwertes ist eine kontinuierlich Größenzunahme von C. suaveolens von Norden nach Süden in 23 Maßen festgestellt worden.

3. Die Unterart balcanica zeigt nach Ondrias (1970) die größten $\mathrm{Maße}$, doch einige vorliegende Exemplare von der Peleponnes bestätigen dies nicht eindeutig, da sie im Mittelwert (CBL) nur mit einem Zehntel Millimeter über dem entsprechenden von Ondrias angegebenem Wert für die Unterart balcanica liegen.

4. Es drängt sich hier die Frage auf, ob eine Diskussion auf Unterartbasis sinnvoll, da hier anscheinend das Maximum eines Klins als eigene Unterart beschrieben worden ist.

5. Der systematische Status von italica und debeauxi bleibt nach wie vor ungeklärt. Die untersuchten Exemplare aus Italien fallen in die Variationsbreite von dalmatinischen und makedonischen suaveolens. Sie sind größer als die wenigen untersuchten Tiere von der Peleponnes.

6. Wenn überhaupt auf Unterartbasis diskutiert werden soll, dann sollte man die Tiere aus BR-Deutschland und Österreich zu mimula rechnen und alle jugoslawischen und griechischen Tiere als balcanica zusammenfassen, mit zunehmendem Größenwachstum nach Süden. Es bleibt dann zu überprüfen, ob die italienischen Exemplare einer gesonderten Subspecies angehören.

Danksagung: Für wertvolle Unterstützung und Ausleihe von Untersuchungsmaterial bin ich Herrn Dr. H. Felt en (Frankfurt), Prof. Dr. E. Von Lehmann (Bonn), Prof. Dr. J. N i e th a mmer (Bonn) und Prof. Dr. H. Heim de Bals a c (Paris) zu größtem Dank verpflichtet.

\section{LITERATUR}

1. Chaworth-Musters J. L., 1932: A contribution to our knowledge of the mammals of Macedonia and Thessaly. Ann. Mag. nat. Hist., London 9, 10: $166-171$.

2. Kahmann H., 1964: Contribution à l'étude des mammifères du Péloponèse. Mammalia, Paris 28: 110-136.

3. Kahmann H. \& V esmanis I., 1975: Morphometrische Untersuchungen an Wimperspitzmäusen (Crocidura), 2. Zur weiteren Kenntnis von Crocidura gueldenstaedti (P all a s 1811) auf der Insel Kreta. Opuscula Zool. 136: 1-12. München.

4. Mille r G. S., 1912: Catalogue of the mammals of Western Europe. Brit. Mus. Nat. Hist.: 1-1019. London.

5. N i eth a m m e r J., 1962: Die Säugetiere von Korfu. Bonn. zool. Beitr., 13, 1-3: $1-49$. 
6. Ondrias J. C., 1970: Contribution to the knowledge of Crocidura suaveolens (Mammalia, Insectivora) from Greece, with a description of a new subspecies Z. f. Säugetierkde., 35: 371-381.

7. Spitzenberger F., 1970: Zur Verbreitung und Systematik türkischer Crociduarinae (Inscectivora, Mammalia). Ann naturhist. Mus. Wien, 74: 233-252.

8. Vesmanis I., 1975: Morphometrische Untersuchungen an algerischen Wimperspitzmäusen, 1. Die Crocidura russula-Gruppe (Mammalia: Insectivora) Senck, biol., 56, 1-3: 1-19.

9. W a g n e r O. S., 1974: Biogeographische Untersuchungen an Kleinsäugerpopulationen des Karpatenbeckens. Inaug. Diss. Saarbrücken: 1-275.

10. W itte G., 1964: Zur Systematik der Insektenfresser des Monte-Gargano Gebiets (Italien). Bonn. zool. Beitr., 15: 1-25.

11. Wolf, in Peus F., 1958: Flöhe aus dem Mittelmeergebiet (Ins. Siphonapt.). II. Griechenland. Mitt. zool. Mus. Berlin, 34: 135-171.

Accepted, May 25, 1976.

Indulis VESMANIS

\section{PORÓWNAWCZE BADANIA MORFOLOGICZNE NAD ZĘBIEŁKIEM KARLICZKIEM W JUGOSEAWII}

Streszczenie

W Jugosławii wystẹpują dwa podgatunki zębiełka karliczka Crocidura suaveolens (P a 11 a s, 1811): mimula w Kroacji i Istrii a balcanica w środkowej i południowej części kraju. Dane (Tabela 1, Ryc. 1-8) wskazują, że dokładne rozdzielenie północno- i srodkowo-jugosłowiańskich od południowojugosłowiańskich $C$. suaveolens jest niemożliwe. Na podstawie wartości średnich dla 23 pomiarúw stwierdzono stopniowy wzrost wielkości zębiełków z N na S.

Podgatunek balcanica wykazuje wg Ondrias a (1970) największe pomiary, jednakże kilka badanych okazów z Peloponezu (Tabela 3) nie potwierdza tego jednoznacznie. Nasuwa się też pytanie, czy w tym przypadku dyskusja przynależności podgatunkowej w ogóle ma sens, ponieważ widocznym jest, że wartości maksymalne $\mathrm{z}$ zakresu zmienności klinalnej zostały opisane jako odrębny podgatunek. Stanowisko systematyczne italica i debauxi pozostaje nadal niewyjaśnione. Badane okazy z Włoch mieszczą się w zakresie zmienności dalmatyńskich i macedońskich C. suaveolens (Tabela 2). Są one większe niż kilka badanych zwierząt z Peloponezu. W kategoriach systematyki podgatunkowej zwierzęta z poludniowej części RFN i Austrii należałoby zaliczyć do mimula, zaś wszystkie jugosłowiańskie i greckie połączyć w balcanica ze wzrastająca wielkością na S. Sprawdzenia. wymaga, czy okazy z Włoch należą do oddzielnego podgatunku. 